\title{
Exploiting Silicon photomultipliers for measuring nonclassical optical states
}

\author{
Giovanni Chesi ${ }^{1, *}$, Alessia Allevi ${ }^{1, * *}$, and Maria Bondani ${ }^{2, * * *}$ \\ ${ }^{1}$ Dipartimento di Scienza e Alta Tecnologia, Università degli Studi dell’Insubria, Via Valleggio 11, I-22100 Como (Italy) \\ ${ }^{2}$ Istituto di Fotonica e Nanotecnologie, IFN-CNR, Via Valleggio 11, I-22100 Como (Italy)
}

\begin{abstract}
The search for optimal receivers endowed with photon-number-resolving capability has led us to consider Silicon photomultipliers for Quantum Optics experiments. By adopting an optimization procedure in order to reduce the spurious effects characterizing these detectors, we succeeded in detecting nonclassical correlations between the two parties of mesoscopic twin-beam states and in obtaining subPoissonian conditional states. These results put SiPMs forward for the implementation of Quantum Information and Communication protocols.
\end{abstract}

\section{Introduction}

Nowadays, most implementations of quantum systems address optical states. Unfortunately, the generation and manipulation of nonclassical states of light are still hard tasks. Nonlinear properties of materials or the emissions of $n$ level systems are usually employed to this aim. However, a basic resource for the generation of quantum states is provided by the very principle of quantum mechanics, in particular by the reduction postulate [1]. Indeed, it is well known that, if a portion of a composite entangled state is measured, the other portion is conditionally reduced according to the exact correlation between the parties [2-6]. The generation of such conditional states is affected by the non-unitary quantum efficiency of the employed detectors. However, other nonidealities could be relevant, depending on which class of detector is used.

The search for sensors endowed with photon-number resolution have led to consider silicon photomultipliers (SiPMs) as a promising choice for quantum optics experiments [7-9]. A SiPM is basically a matrix of single-photon avalanche diodes, working in Geiger-Muller regime, which are called cells or pixels [10-14]. Besides their outstanding photon-number-resolving capability, SiPMs work at room temperature and low bias voltage. Moreover, they are ideal for compact systems. Anyway, they are also affected by some spurious effects which could be critical for quantum correlation measurements. The most relevant of them are known as dark counts, optical cross talk and afterpulses [15-17]. Dark counts consist of spurious avalanches triggered by thermally generated charge carriers, while optical cross-talk is the emission of a Bremsstrahlung photon by a photoelectron, which fires a neighbouring cell thus yielding a spurious count. Finally, the geometric imperfections of the device struc-

\footnotetext{
*e-mail: gchesi@uninsubria.it

**e-mail: alessia.allevi@uninsubria.it

***e-mail: maria.bondani@uninsubria.it
}

ture can capture photoelectrons that, when released, start new avalanches, called afterpulses.. In our previous works $[18,23]$, we have shown that, for what concerns the last generation of SiPMs, the cross-talk probability has been consistently reduced and the afterpulses has been made negligible. Thanks to these improvements, we have recently demonstrated that SiPMs can be used to reveal the nonclassicality of mesoscopic twin-beam states of light and to produce subPoissonian states by means of conditional measurements [18, 19].

In Sect. 2 we briefly introduce the model describing the light detection with SiPMs. Then, in Sect.3 we present the experimental results concerning the characterization of the nonclassical nature of mesoscopic twin-beam states and the generation of subPoissonian conditional states. Finally, in Sect. 4 we draw our conclusions and discuss some possible future improvements.

\section{Photodetection model}

The general model typically adopted to describe the statistics of the light detected by the new generation of SiPMs includes the detection process due to a non-ideal detection efficiency, the presence of dark counts and the cross-talk effect.

If $\mathrm{P}_{n}$ is the probability that the light state $\hat{\rho}$ is in the $n$-th eigenstate of the Fock basis

$$
\mathrm{P}_{n} \equiv\langle n|\hat{\rho}| n\rangle
$$

and $\mathrm{p}_{k}$ is the probability of detecting $k$ photoelectrons, the relation between the two distributions is given by

$$
\mathrm{p}_{k}=M_{n}^{(k)} \mathrm{P}_{n}
$$

where $M_{n}^{(k)}$ is the probability to detect $k$ photons when a $n$-photon Fock state impinges on the device [20]. For those three contributions, i.e. the non-unitary quantum 
efficiency, dark-count and cross-talk effects, it has been shown [7, 21, 22] that the transformations $M_{n}^{(k)}$ are linear. The probabilities $M_{n}^{(k)}$ are elements of a matrix $\mathbf{M}$ given by the convolution of the three mentioned stochastic processes. In the case of a non-unitary quantum efficiency $\eta$, the process is Bernoullian, i.e.

$$
\mathrm{p}_{m}=\eta^{m} \sum_{n=m}^{\infty}\left(\begin{array}{l}
n \\
m
\end{array}\right)(1-\eta)^{n-m} \mathrm{P}_{n},
$$

whose mean value and variance are

$$
\begin{aligned}
& \langle m\rangle=\eta\langle n\rangle \\
& \sigma_{m}^{2}=\eta^{2} \sigma_{n}^{2}+\eta(1-\eta)\langle n\rangle .
\end{aligned}
$$

Since dark counts are independent events, their statistical distribution is Poissonian, namely

$$
P_{\mathrm{dc}}(m)=\frac{\left(\langle m\rangle_{\mathrm{dc}}\right)^{m}}{m !} \exp \left[-\langle m\rangle_{\mathrm{dc}}\right] .
$$

Convolving Eq. (2) with Eq. (4) we get

$$
\mathrm{p}_{\tilde{m}}=\sum_{m=0}^{\tilde{m}} \eta^{m} \sum_{n=m}^{\infty}\left(\begin{array}{l}
n \\
m
\end{array}\right)(1-\eta)^{n-m} \frac{\left\langle m_{d c}\right)^{\tilde{m}-m}}{(\tilde{m}-m) !} \exp \left(-\left\langle m_{d c}\right\rangle\right) \mathrm{P}_{n}
$$

This contribution is responsible for a shift of a quantity $\left\langle m_{\mathrm{dc}}\right\rangle$ of the mean value and the variance in Eq. (3), namely

$$
\begin{aligned}
& \langle\tilde{m}\rangle=\langle m\rangle+\left\langle m_{d c}\right\rangle \\
& \sigma_{\tilde{m}}^{2}=\sigma_{m}^{2}+\left\langle m_{d c}\right\rangle .
\end{aligned}
$$

The cross-talk effect acts in the opposite sense with respect to the quantum efficiency, the latter reducing the number of counted photons with a non-unit success probability $\eta$, the former enhancing it with probability $\varepsilon$ [7]. Thus, we are left with a Binomial-like contribution

$$
C_{k, l}(\varepsilon)=\left(\begin{array}{c}
l \\
k-l
\end{array}\right) \varepsilon^{k-l}(1-\varepsilon)^{2 l-k}
$$

leading to

$$
\begin{aligned}
\mathrm{p}_{k}= & \sum_{\tilde{m}=k / 2}^{k}\left(\begin{array}{c}
\tilde{m} \\
k-\tilde{m}
\end{array}\right) \varepsilon^{k-\tilde{m}}(1-\varepsilon)^{2 \tilde{m}-k} \sum_{m=0}^{\tilde{m}} \eta^{m} \sum_{n=m}^{\infty}\left(\begin{array}{l}
n \\
m
\end{array}\right)(1-\eta)^{n-m} \\
& \frac{\left\langle m_{d c} c^{\tilde{m}-m}\right.}{(\tilde{m}-m) !} \exp \left(-\left\langle m_{d c}\right\rangle\right) \mathrm{P}_{n},
\end{aligned}
$$

whose mean value and variance read

$$
\begin{aligned}
\langle k\rangle & =(1+\epsilon)\langle\tilde{m}\rangle \\
\sigma_{k}^{2} & =(1+\epsilon)^{2} \sigma_{\tilde{m}}^{2}+\epsilon(1-\epsilon)\langle\tilde{m}\rangle .
\end{aligned}
$$

\section{Experimental results}

The experimental setup used to test if SiPMs can properly reveal the nonclassicality of quantum states is shown in Fig. 1. The fourth harmonic (at $262 \mathrm{~nm}$ ) pulses of a $\mathrm{Nd}$ :YLF laser regeneratively amplified at $500 \mathrm{~Hz}$ are generated by sum-frequency generation in a $\beta$-barium-borate crystal (BBO1) and sent to a second $\mathrm{BBO}(\mathrm{BBO} 2)$ in order

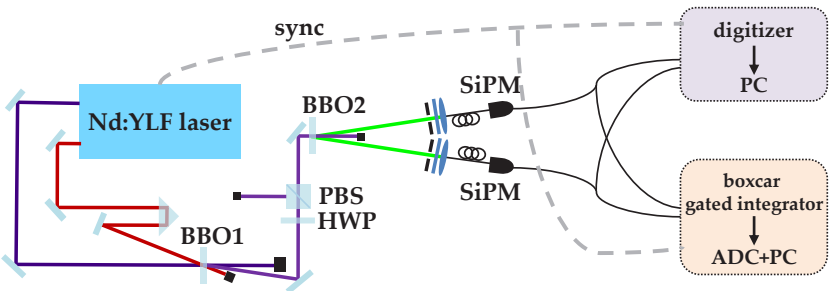

Figure 1. Sketch of the experimental setup. See the text for details.

to produce parametric downconversion. Two twin portions of the obtained twin-beam state are spatially and spectrally selected at frequency degeneracy (at $523 \mathrm{~nm}$ ) by means of two irises and interference filters, respectively, and delivered by a pair of multi-mode fibers to two SiPMs (mod. MPPC S13360-1350CS, Hamamatsu Photonics). Each detector output is then amplified and synchronously integrated by a boxcar-gated integrator (SR250, Stanford Research Systems). The state characterization is performed as a function of pump intensity, which is modified through a half-wave plate (HWP) followed by a polarizing cube beam splitter (PBS). For each energy value, $10^{5}$ singleshot acquisitions are performed.

As already demonstrated in Ref. [23], in order to reduce the effect of the nonidealities described in Sect. 2, a proper integration of the signal must be performed. In particular, the cleanest information on the detected light is contained in the peak of the electronic output signal. That is why, in the following we show the experimental results achieved by integrating the signal over $10 \mathrm{~ns}$, which represents the minimum value compatible with the measurement stability [18]. Under this condition, both the dark counts and the cross-talk effect can be considered negligible. That is why in the following we are not considering them anymore. In order to quantify the nonclassicality of the generated twin-beam states, one of the most used criteria is given in terms of the so-called noise reduction factor $R$, which is defined as

$$
R(n)=\frac{\sigma^{2}\left(n_{1}-n_{2}\right)}{\left\langle n_{1}+n_{2}\right\rangle} .
$$

where $\sigma^{2}\left(n_{1}-n_{2}\right)$ is the variance of the photon-number difference in the two twin arms, while $\left\langle n_{1}+n_{2}\right\rangle$ is the shotnoise level. Values of $R$ lower than 1 are sufficient to prove the nonclassicality of the state under examination. The expression in Eq. (9) can be written also in terms of measurable quantities. For instance, it is useful to rewrite it in terms of detected photons, i.e.

$$
R(m)=\frac{\sigma^{2}\left(m_{1}-m_{2}\right)}{\left\langle m_{1}+m_{2}\right\rangle},
$$

In Ref. [19] we have shown that an alternative nonclassicality criterion can be expressed also by means of Glauber's second-order autocorrelation functions, i.e.

$$
g^{(2)}(n) \equiv \frac{\left\langle n^{2}\right\rangle}{\langle n\rangle^{2}}=\frac{\sigma_{n}^{2}}{\langle n\rangle^{2}}+1 .
$$


Indeed, it is possible to demonstrate that the second-order autocorrelation function of the photon-number difference

$$
g_{\text {diff }}^{(2)}(n)=\frac{\left\langle\left(n_{1}-n_{2}\right)^{2}\right\rangle}{\left\langle n_{1}-n_{2}\right\rangle^{2}}
$$

is linked to the noise reduction factor as follows

$$
g_{\mathrm{diff}}^{(2)}(n)-1=[R(m)-1] \frac{\left\langle m_{1}+m_{2}\right\rangle}{\left\langle m_{1}-m_{2}\right\rangle^{2}} .
$$

According to this equation, the negativity of the quantity $\left[g_{\text {diff }}^{(2)}(n)-1\right]$ certifies the nonclassicality of the state as well as the condition $R<1$.

In Fig. 2 we show the values of $\left[g_{\text {diff }}^{(2)}(n)-1\right]$ calculated from the experimental data as black dots and error bars. Note that all the presented data are negative, thus proving that SiPMs can reveal the nonclassical nature of the produced twin-beam states. The data are well superimposed to the theoretical expectation calculated according to the definition in Eq. (12) in which we substituted the experimental values of $\left\langle m_{1}\right\rangle$ and $\left\langle m_{2}\right\rangle$, whereas $R$ was evaluated according to the model valid for multi-mode twin-beam states, that is

$$
R=1-\frac{2 \eta \sqrt{\left\langle m_{1}\right\rangle\left\langle m_{2}\right\rangle}}{\left\langle m_{1}\right\rangle+\left\langle m_{2}\right\rangle}+\frac{\left(\left\langle m_{1}\right\rangle-\left\langle m_{2}\right\rangle\right)^{2}}{\mu\left(\left\langle m_{1}\right\rangle+\left\langle m_{2}\right\rangle\right)}
$$

for equal number of modes $\mu$ and detection efficiency $\eta$ in the two arms.

As anticipated in the Introduction, the application of con-

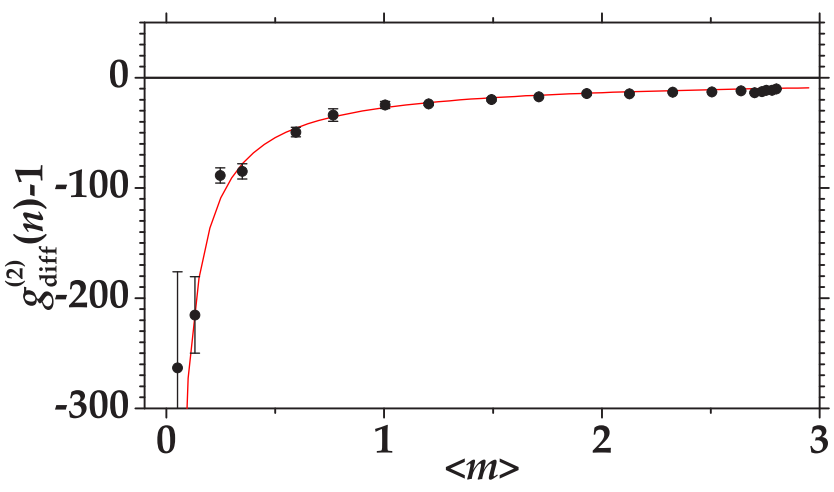

Figure 2. Measured $\left[g_{\text {diff }}^{(2)}(n)-1\right]$ as a function of $\langle m\rangle=\left(\left\langle m_{1}\right\rangle+\right.$ $\left.\left\langle m_{2}\right\rangle\right) / 2$. Black dots and error bars: experimental data; red line: theoretical expectation according to Eq. (12), in which $R$ was evaluated according to Eq. (13).

ditional measurements to entangled states, such as twin beams, yields nonclassical states of light. In the presence of an ideal detection process, the conditional states reduce to Fock states, being the twin beam equally populated in the two twin arms. However, in the case of nonideal detection, only subPoissonian states can be obtained. In some previous works $[1,5]$ we have demonstrated that such subPoissonian states have a mean number of photons $\langle m\rangle_{\text {cond }}$ depending on the conditioning value $m_{\text {cond }}$, namely the number of photons measured in one arm according to which the data in the other arm are selected. If the unconditioned state is multi-mode thermal, the expression of $\langle m\rangle_{\text {cond }}$ is analytic and reads as

$$
\langle m\rangle_{\mathrm{cond}}=\frac{(\langle m\rangle+\eta \mu)}{\langle m\rangle+\mu} m_{\mathrm{cond}}+\frac{\mu\langle m\rangle(1-\eta)}{\langle m\rangle+\mu}
$$

The mean number of photons of the conditional states as a function of the conditioning value starting from a twin beam with $\langle m\rangle=2.52$ is shown in Fig. 3. The data are shown as black dots with error bars, while the theoretical expectation according to Eq. (14) is presented as a red curve. In order to quantify the nonclassical nature of the

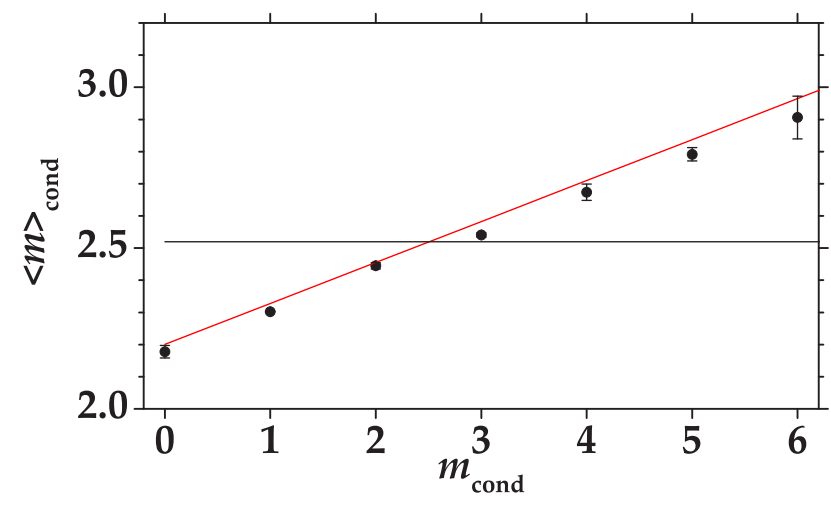

Figure 3. Mean number of photons of the conditional states as a function of the conditioning value. Black dots and error bars: experimental data; red line: theoretical expectation according to Eq. (14). The black straight line indicates the mean number of photons of the unconditioned state, namely $\langle m\rangle=2.52$.

conditional states achieved so far, it is useful to evaluate their Fano factor. In particular, its expression for detected photons is given by [5]

$$
\begin{aligned}
& F_{\text {cond }}=(1-\eta)+(1-\eta) \\
& \times \frac{\langle m\rangle\left(m_{\text {cond }}+\mu\right)(\langle m\rangle+\eta \mu)}{(\langle m\rangle+\mu)\left[\left(m_{\text {cond }}+\mu\right)(\langle m\rangle+\eta \mu)-\eta \mu(\langle m\rangle+\mu)\right]}
\end{aligned}
$$

We displayed in Fig. 4 the calculated values of Fano factor as a function of the conditionig value for the conditional states presented in Fig. 3. The experimental data are shown as black dots and error bars, while the theoretical expectation, plotted according to Eq. (15), as a red curve. We note that, except for the case $m_{\text {cond }}=0$, corresponding to a Gaussian operation, the values of $\mathrm{F}$ are all below 1, thus proving the subPoissonian nature of the conditional states. The horizontal line in the figure refers to the value of the Fano factor of the unconditioned state, namely $F=1.000126$. In general, we note that the larger the conditioning value, the more subPoissonian the state. We also remark that, at variance with other works concerning the production of conditional states, the use of SiPMs guarantees the possibility to generate well-populated conditional states. 


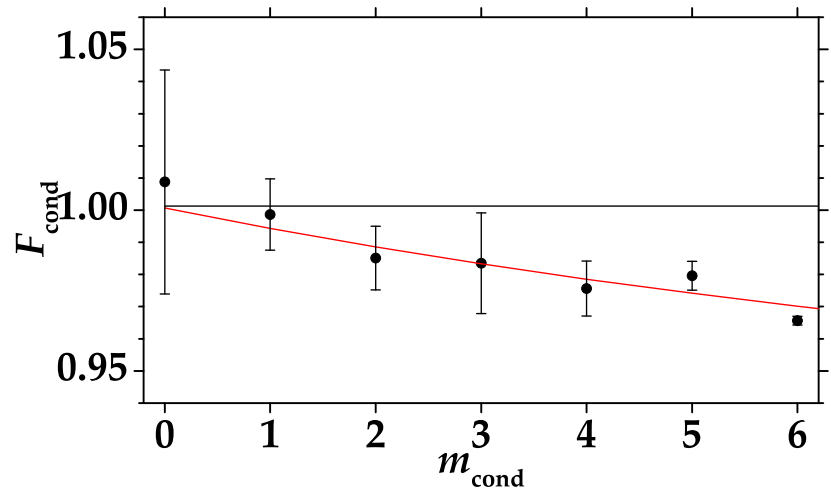

Figure 4. Fano factor of the conditional states as a function of the conditioning value. Black dots and error bars: experimental data; red line: theoretical expectation according to Eq. (15). The horizontal black line is at the value of the Fano factor of the unconditioned state, namely $F=1.000126$.

\section{Conclusions}

In conclusion, we have demonstrated that the new generation of SiPMs, properly optimized, can be successfully used to reveal nonclassicality of quantum states of light. In particular, we have shown that they can detect sub-shot-noise photon-number correlations of mesoscopic twin-beam states and that can be used to perform conditional measurements and produce subPoissonian states. Even if the global quantum efficiency of the detection system is not so high, there is still room for improvement, especially for what concerns the input and output coupling of light into the fibers. The main remarkable result is given by the explored dynamic range, which involved well-populated states of light, thus proving the usefulness of SiPMs for state characterization in the very mesoscopic regime.

\section{References}

[1] A. Allevi, A. Andreoni, F. A. Beduini, M. Bondani, M. G. Genoni, S. Olivares, M. G. A. Paris, Europhys. Lett. 92, 6 (2010).

[2] J. Laurat, T. Coudreau, N. Treps, A. Maĭtre, C. Fabre, Phys. Rev. Lett. 91, 213601 (2003).

[3] J. Laurat, T. Coudreau, N. Treps, A. Maiitre, C. Fabre, Phys. Rev. A 69, 033808 (2004).
[4] A. Ourjoumtsev, R. Tualle-Brouri, P. Grangier, Phys. Rev. Lett. 96, 213601 (2006).

[5] M. Lamperti, A. Allevi, M. Bondani, R. Machulka, V. Michálek, O. Haderka, J. Peřina Jr., J. Opt. Soc. Am. B 31, 5 (2014).

[6] T. S. Iskhakov, V. C. Usenko, U. L. Andersen, R. Filip, M. V. Chekhova, G. Leuchs, Opt. Lett. 41, 2149 (2016).

[7] I. Afek, A. Natan, O. Ambar, Y. Silberberg, Phys. Rev. A 79, 6 (2009).

[8] M. Ramilli, A. Allevi, V. Chmill, M. Bondani, M. Caccia, A. Andreoni, J. Opt. Soc. Am. B 27, 10 (2010).

[9] D. A. Kalashnikov, S. H. Tan, T. S. Iskhakov, M V. Chekhova, L. A. Krivitsky, Opt. Lett. 37, 2829 (2012).

[10] A. V. Akindinov, A N. Martemianov, P. A. Polozov, V. M. Golovin, E. A. Grigoriev, Nucl. Instrum. Methods Phys. Res. A 387, 231 (1997).

[11] G. Bondarenko, B. Dolgoshein, V. Golovin, A. Ilyin, R. Klanner, E. Popovaa, Nucl. Physics. B (proc. Suppl.) 61B, 347-352 (1998).

[12] V. Saveliev, V. Golutvin, Nucl. Instrum. Methods A 442, 223 (2000).

[13] C. Piemonte, Nucl. Instrum. Methods A 568, 224 (2006).

[14] D. Renker, E. Lorenz, J. Instrum. 4, P04004 (2009).

[15] A. Gola, A. Ferri, A. Tarolli, N. Zorzi, C. Piemonte, Phys. Med. Biol. 59, 3615-3635 (2014).

[16] Y. Du, F. Retière, Nucl. Instrum. Methods Phys. Res. A 596, 396-401 (2008).

[17] F. Nagy, M. Mazzillo, L. Renna, G. Valvo, D. Sanfilippo, B. Carbone, A. Piana, G. Fallica, J. Molnár, Nucl. Instrum. Methods Phys. Res. A 759, 44-49 (2014).

[18] G. Chesi, L. Malinverno, A. Allevi, R. Santoro, M. Caccia, M. Bondani, Opt. Lett. 44, 1371-1374 (2019).

[19] G. Chesi, A. Allevi, M. Bondani, Quantum Meas. Quantum Metrol. 6, 1-6 (2019).

[20] G. Chesi, A. Allevi, M. Bondani, to appear in Int. J. Quantum Inform. (2019).

[21] D. Achilles, C. Silberhorn, C. Sliwa, K. Banaszek, I. A. Walmsley, M. J. Fitch, B. C. Jacobs, T. B. Pittman, J. D. Franson, J. Mod. Opt. 51, 16 (2004).

[22] M. J. Fitch, B. C. Jacobs, T. B. Pittman, J. D. Franson, Phys. Rev. A 68, 6 (2003).

[23] G. Chesi, L. Malinverno, A. Allevi, R. Santoro, M. Caccia, A. Martemiyanov, M. Bondani, Sci. Rep. 44, 9 7433 (2019). 